\title{
Langfristverordnungen bei Heilmitteln: Kassenblockade brechen!
}

- Nach dem GKV-Versorgungsstrukturgesetz (GKV-VStG) und den Heilmittelrichtlinien können Verordnungen außerhalb des Regelfalls, insbesondere längerfristige Verordnungen getätigt werden, wenn sich die Behandlung eines Krankheitsbildes nach den Vorgaben des Heilmittelkataloges nicht mit einer bestimmten Gesamtverordnungsmenge abschließen lässt.

Auf Antrag der Patienten können derartige längerfristige Verordnungen, wenn sich ihre Notwendigkeit aus einer ärztlichen Begründung ergibt, von der Krankenkasse langfristig genehmigt werden. Langfristig genehmigte Heilmittelverordnungen auBerhalb des Regelfalls werden aus dem Heilmittelbudget herausgerechnet und unterliegen nicht der Richtgrößenprüfung (§ 32 SGB V). Ablehnen können die Krankenkassen einen derartigen Antrag des Patienten nur aus medizinischen Gründen. Dies bedeutet, dass aufgrund der Ablehnung durch die Krankenkasse eine vom Arzt als notwendig erachtete lang anhaltende und kontinuierliche Heilmitteltherapie von den Krankenkassen als medizinisch nicht erforderlich und unwirtschaftlich angesehen wird und folgerichtig der Arzt die Notwendigkeit der kontinuierlichen Heilmitteltherapie bei diesen Patienten überprüfen und ggf. reduzieren muss.

Der Patient beantragt die Langfristgenehmigung indem er die korrekt und vollständig ausgefüllte Heilmittelverordnung außerhalb des Regelfalls inklusive der erforderlichen prognostischen Begründung zusammen mit einer vom Arzt ausgefüllten ärztlichen Bescheinigung bei seiner Krankenkasse vorlegt. Die ärztliche Bescheinigung attestiert dabei die Notwendigkeit der Behandlung außerhalb des Regelfalls. Damit verfügen die Krankenkassen über alle Informationen, die sie für die Ausstellung einer derartigen Genehmigung benötigen.

\section{MMW Kommentar}

Obwohl die Patienten seit dem 1.1.2012 mit dem GKV-VStG das Recht auf eine Genehmigung ihrer Heilmittelverordnung außerhalb des Regelfalls als Langfristverordnung haben, wird dieses Verfahren von vielen Krankenkassen noch nicht umgesetzt bzw. durch umfangreiche Rückfragen an den Patienten bzw. die verordnenden Ärzte erschwert bzw. behindert.

Diese Rückfragen fordern auch Leistungsoder Dokumentationsinhalte an, die es als EBM-Leistung überhaupt nicht gibt. Grundsätzlich ist hierzu festzuhalten, dass derart umfangreiche Anfragen nicht den vertraglichen Bestimmungen der Vordruckvereinbarung entsprechen, in der Anfragen zu Behandlungen und Arzneimitteltherapien der Kassen an die Ärzte geregelt sind. Die Kassen dürfen zwar in Ausnahmefällen Auskünfte auf nicht vereinbarten Vordrucken anfordern, müssen dann aber die Rechtsgrundlage für die Auskunftspflicht angeben und im Einzelfall nachweisen, warum sie diese Auskunft benötigen. Fehlt dieser Hinweis, ist der Vertragsarzt nicht verpflichtet, die Anfrage zu beantworten. Gleiches gilt wenn die Anfrage der Kasse wie in diesen Fällen, über ein einfaches Auskunftsbegehren hinausgeht, ohne dass dabei ein vereinbarter Vordruck der Vordruckvereinbarung verwendet wird. Bezüglich solcher Fälle vertritt die KBV die Auffassung, dass diese Auskünfte nicht nach den Leistungspositionen des EBM berechnet werden können.

Derartige Anfragen können entweder mit Verweis auf die Rechtslage unbeantwortet zurückzugeben oder nach vorheriger Kostenzusage durch die Krankenkasse privat nach GOÄ liquidiert werden. Hinzu kommt, dass es sich bei dieser nicht vertragskonformen Auskunftsanforderung um die Weitergabe von Patientendaten an Dritte außerhalb der gesetzlichen Pflichten eines Vertragsarztes handelt, sodass der Vertragsarzt den betroffenen Patienten darüber informieren muss. Auch eine Langfristverordnung wird als Verordnung außerhalb des Regelfalls durchgeführt und quartalsweise nach der erforderlichen Kontrolluntersuchung ausgestellt. Lehnt die Krankenkasse eine Langfristverordnung ab, erhält der Patient einen entsprechenden Bescheid, gegen den er Widerspruch einlegen kann.

\section{Neue Dokumentationssoftware für DMP Asthma}

\footnotetext{
— Der G-BA hat am 24.5.2012 die Richtlinie zur Ausgestaltung der Disease-Management-Programme Brustkrebs, Asthma und COPD endgültig beschlossen. Die Inkraftsetzung der Richtlinie durch deren Veröffentlichung im Bundesanzeiger wird innerhalb der nächsten vier Wochen erwartet. Die aktualisierten Anforderungen betreffen die Dokumentation der Programme Brustkrebs und Asthma; beim COPD bleibt die Dokumentation unverändert.
}

\section{MMW Kommentar}

Die Software-Anbieter wurden seitens der Kassenärztlichen Bundesvereinigung (KBV) bereits aufgefordert, ihre Software-Produkte auf die geänderten Datensätze und Plausibilitäten hin anzupassen. Die technische Realisierung der Änderungen für den Praxisbetrieb wird zum Stichtag 1. Januar 2013 angestrebt. 